\title{
Sprache, Grammatik, Variation - viticulture linguistique
}

Stark, Elisabeth

\begin{abstract}
The aim of this contribution is to reposition a key notion to the centre of Wulf Oesterreicher's theoretical disciplinary reasoning: grammar, or more generally, 'rules and norms' specific to single languages and varieties the linguist deals with. This includes contingent, non-motivated structures, and claiming the necessity to analyse their existence 'as such' (i.e., also independently of communicative, social etc. factors) as the core task of linguistics. This point is illustrated with a much debated variable in French descriptive linguistics: past participle agreement. Combining theoretical and corpus linguistic observations, the distribution of the two variants (absence vs. realization of agreement) in constructions with the auxiliary avoir shows that a grammatical analysis interested in the derivation of certain rules and their application in actual language usage permits to understand the logic of linguistic variation - at least the one observable alongside the standard - non-standard opposition in French.
\end{abstract}

DOI: https://doi.org/10.1515/roja-2018-0004

Posted at the Zurich Open Repository and Archive, University of Zurich

ZORA URL: https://doi.org/10.5167/uzh-159658

Journal Article

Published Version

The following work is licensed under a Publisher License.

Originally published at:

Stark, Elisabeth (2018). Sprache, Grammatik, Variation - viticulture linguistique. Romanistisches Jahrbuch, 69:96-118.

DOI: https://doi.org/10.1515/roja-2018-0004 


\title{
Elisabeth Stark (Zürich) \\ Sprache, Grammatik, Variation - viticulture linguistique
}

\begin{abstract}
The aim of this contribution is to reposition a key notion to the centre of Wulf Oesterreicher's theoretical disciplinary reasoning: grammar, or more generally, 'rules and norms' specific to single languages and varieties the linguist deals with. This includes contingent, non-motivated structures, and claiming the necessity to analyse their existence 'as such' (i.e., also independently of communicative, social etc. factors) as the core task of linguistics. This point is illustrated with a much debated variable in French descriptive linguistics: past participle agreement. Combining theoretical and corpus linguistic observations, the distribution of the two variants (absence vs. realization of agreement) in constructions with the auxiliary avoir shows that a grammatical analysis interested in the derivation of certain rules and their application in actual language usage permits to understand the logic of linguistic variation - at least the one observable alongside the standard - non-standard opposition in French.
\end{abstract}

\section{Einleitung}

Der unmittelbare Anlass für die folgenden Überlegungen war die traurige Nachricht, nach dem Tode Peter Kochs 2014 etwas mehr als ein Jahr später auch Wulf Oesterreicher verloren $\mathrm{zu}$ haben. Im Kontext des Gedenkens und Trauerns entstand sofort ein fruchtbarer Boden ${ }^{1}$, um insbesondere ihre sprachtheoretischen Überlegungen erneut zu reflektieren, in ihrem Ursprung, ihrer genuinen Intention, aber auch in ihrem Wirken weit über die deutschsprachige Romanistik hinaus (s. dazu die Beiträge in Feilke/Hennig 2016 und insbesondere Selig 2017). Die tieferliegende Motivation, ausgehend von Coseriu (z.B. Coseriu 1988), Söll (z.B. Söll $\left.{ }^{3} 1985\right)$ und schließlich Wulf Oesterreichers Schriften nach dem Kern des sprachwissenschaftlichen Arbeitens zu fragen, was im Folgenden geschehen soll, stammt aber von einem Unbehagen her, das ich in aller Unbescheidenheit mit Wulf Oesterreicher und anderen geschätzten Kollegen der (romanistischen)

1 Dieser Beitrag stellt die überarbeitete Version meines Vortrags anlässlich der Gedenkfeier für Wulf Oesterreicher am 26.5.2016 an der Ludwig-Maximilians-Universität München dar. Wesentliche Anregungen dafür stammen von Andreas Dufter, Daniel Jacob, Wolf-Dieter Stempel und insbesondere Maria Selig, welchen ich dafür zu herzlichem Dank verpflichtet bin. 
Sprachwissenschaft $\mathrm{zu}$ teilen meine und das man mit der Wahrnehmung, disziplinärer Unschärfe‘ und manchmal auch theoretischer und methodischer Sorglosigkeit umschreiben könnte. Dieses Unbehagen hängt mit der Existenz und dem immer häufigeren Gebrauch von meines Erachtens nach pleonastischen Komposita wie Systemlinguistik zusammen, die mittlerweile nicht selten nur eine kleine Minderheit von Inhalten an kleineren wie größeren sprachwissenschaftlichen Veranstaltungen oder linguistischen und philologischen Curricula von allem anderen absetzen. Dabei geht es nicht um ein bildungs- oder forschungspolitisches Fanal, sondern um das Bemühen, einen Bereich, der v.a. in der späteren Rezeption und in den späteren Schriften von Wulf Oesterreicher scheinbar etwas marginalisiert wurde, in seiner Relevanz für die Linguistik und eben auch für die Koch/Oesterreichersche Varietätenlinguistik wieder als zentralen zu benennen und $\mathrm{zu}$ betrachten: die Grammatik ${ }^{2}$, verstanden im Folgenden als Gesamtheit der nicht-lexikalischen Regularitäten auf Satzebene ${ }^{3}$. Es ist vollkommen zutreffend, wenn Maria Selig $(2017,128)$ feststellt, dass „Peter Koch und Wulf Oesterreicher sich klar einer am historischen Sprachwissen orientierten systemischen Linguistik zurechnen“. Viele, wenn nicht alle der also zentral zu beobachtenden Regularitäten sind genuin sprachlich, der menschlichen Sprache zugehörig, was diese von anderen Kommunikationssystemen stark, manche würden sagen kategorisch unterscheidet, und dennoch unterliegen diese Regularitäten wie das Lexikon ohne Zweifel der der menschlichen Sprache ebenfalls definitorisch eigenen Variation und der Abhängigkeit von kognitiven und kommunikativinteraktiven Bedingungen ${ }^{4}$. So erklärt sich die Anordnung der im Folgenden

2 D.h. ich setze einen anderen Akzent als Maria Selig in ihrem kürzlich erschienenen sehr einsichtsreichen Jahrbuch-Beitrag (cf. Selig 2017), der sich intensiv mit dem Koch/Oesterreicherschen Nähe-Distanz-Kontinuum als „Theoretisierung der situativ-sprachlichen Variation“ (Selig 2017, 123) und seiner v.a. germanistischen Rezeption auseinandersetzt. Die dortige Argumentation ist in ihrer Grundanlage und häufig auch im Detail völlig überzeugend; wo sich mir Fragen stellen und dies im Zusammenhang mit meiner Argumentation steht, werde ich detaillierter darauf eingehen.

3 Dies v.a. im Sinne von Oesterreichers (1996a) Begriff der Morphosyntax als „syntagmatischer Sprachzeichenbildung“ (Oesterreicher 1996a, 273), plus der Phonologie, im Folgenden nicht weiter berücksichtigt.

4 Man vergleiche genau in diesem Sinne den zentralen Begriff der Schnittstelle, engl. interface, zu anderen kognitiven und handlungssteuernden menschlichen Systemen, aus deren Eigenschaften mindestens in neueren Versionen der generativen Grammatik (,Minimalist Program“) grundlegende Eigenschaften menschlicher Sprache resultieren (cf. z.B. Chomsky 2005). D.h. die menschliche Sprache wird hier grundsätzlich nicht als hermetisch abgeschlossenes System oder „immanentistisch“ konstruiert (cf. Hauser/Chomsky/Fitch 2002), allerdings, in guter strukturalistischer Tradition, durchaus auch unter Absehung von kommunikativen Bedingungen untersucht. S. als prominente Gegenbeispiele die allermeisten Arbeiten zur linken Peripherie spätestens seit 
wesentlichen drei Begriffe im Titel dieses Beitrags. In Abschnitt 2 werde ich grundsätzliche Aussagen Wulf Oesterreichers zum zentralen Gegenstand der Sprachwissenschaft zusammenfassen, zu denen nach meiner Lesart auch das Erkennen der „Kontingenz“ einzelsprachlicher Regeln und Normen im Sinne von ,nicht kommunikativ bedingt“ wesentlich gehört. Danach wird in Abschnitt 3 die Oesterreichersche „Konstruktion von Varietäten“ reflektiert und nach dem Raum bzw. dem Stellenwert einer gründlichen sprachwissenschaftlichen Untersuchung der den Varietäten eigenen und eben durchaus auch kontingenten Regel- und Normgefügen gefragt, der nach meinem Dafürhalten durchaus größer sein könnte in der aktuellen (romanistischen) Linguistik. Anhand einer Variable mit zwei unterschiedlichen Varianten (binär: An- bzw. Abwesenheit im Standard vs. „français de l'immédiat“), nämlich des accord du participe passé in Konstruktionen mit dem Auxiliar avoir (zur Beschreibung der Regularität s. 3.1) wird anhand einer Korpusanalyse in Abschnitt 3.2, in Kombination mit Erkenntnissen sprachtheoretischer Natur zur Kongruenz (s. dazu auch Stark 2017), die Frage nach dem Warum (nicht: dem Wozu, s. dazu Coseriu 1974) gestellt, also die Frage nach den Ursachen der beobachteten Variation. Diese liegen in den grammatischen Regeln selbst bzw. in ihrer Vereinbarkeit (oder Abweichung) von in menschlichen Sprachen möglichen Kongruenzmustern, womit die beobachtete Verteilung der Varianten auf einmal nicht mehr kontingent, sondern motiviert ist - grammatisch motiviert.

\section{Der paradigmatische Kern der Linguistik}

Ausgangspunkt ist Wulf Oesterreichers vollkommen zutreffende und gleichzeitig angesichts der Ausdifferenzierung der Sprachwissenschaft in den letzten Jahrzehnten sehr radikale Rückbesinnung unserer Disziplin auf den „paradigmatische[n] Kern der Linguistik“ (Oesterreicher 2010b, 79), nämlich die wissenschaftliche Untersuchung von Einzelsprachen und ihren Varietäten als solche. Diese Bestimmung des zentralen Gegenstandsbereichs der Sprachwissenschaft folgt dem Coseriuschen Drei-Ebenen-Modell, das neben dieser historischen Ebene auch eine universale der menschlichen Sprachfähigkeit und eine individuelle des konkreten Diskurses und Textes kennt (cf. Coseriu 21981):

Rizzi 1997 (s. dazu Seiler 2015, 244) oder etwa Liliane Haegemans (z.B. Haegeman 1997, 2013) Publikationen zur Subjektauslassung in Tagebüchern, die die Diskurstradition des Tagebuchs und v.a. ihre spezifisch-kommunikativen Bedingungen bzw. Einschränkungen (Monologizität im Koch/Oesterreicherschen Faktorenbündel zur Modellierung des Nähe-Distanz-Kontinuums) als notwendigen Ausgangspunkt des syntaktischen Erklärungsansatzes nimmt. 
Universelle Ebene:

Historische Ebene:

Aktuelle Ebene:
Sprechtätigkeit [A]

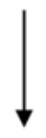

Einzelsprache [B 1]

Diskurstradition [B 2]

Abb. 1: Das Coseriusche Drei-Ebenen-Modell (cf. z.B. Coseriu ${ }^{2} 1981$, zitiert nach Oesterreicher 2010b, 85)

Als „Herzstück der Sprachwissenschaft“ bezeichnet Oesterreicher am gleichen Ort „Sprachliches qua sprachliche[r] Techniken“ (Oesterreicher 2010b, 89), die er, so meine Lesart seiner verschiedenen neueren Stellungnahmen zu diesem Punkt, mit Coseriu und Saussure als sozial geteilte, auch kommunikativ-diskursiv geformte und dem Wandel unterworfene „Regel und Normgefüge“ (Oesterreicher 2010b, 89) ansieht:

Wichtig ist, dass dabei die Sprachen, Idiome und Varietäten immer als Resultate kontingenter einzelsprachlicher und kommunikativ-diskursiver Entwicklungen mit ihren Elementen, Regeln und Normen ein verallgemeinertes soziales Wissen darstellen, das erstens Variation aufweist und zweitens per definitionem historisch fortbildbar, also grundsätzlich geschichtlicher Veränderung unterworfen ist. (Oesterreicher 2015, 23)

$\mathrm{Zu}$ beachten ist im obigen Zitat meines Erachtens die Koordination von zwei Faktoren sprachlicher Veränderlichkeit: „kontingente[r] einzelsprachliche[r] und kommunikativ-diskursive[r] Entwicklungen“. Das scheint im Denken Wulf Oesterreichers nicht identisch zu sein, anders als in manchen neueren Ausprägungen sogenannter gebrauchsbasierter Zugänge zur Sprachbeschreibung. Obwohl, dies steht außer Zweifel, seit seiner Dissertation (cf. Oesterreicher 1979) Wulf Oesterreichers sprachwissenbasierter Sprachbegriff in der menschlichen Sprechtätigkeit gründet (cf. Selig 2017, 124), ist das Merkmal der synchronen und diachronen Kontingenz der Ebene des Historischen ein zentrales, ja definitorisches in seinen Überlegungen, das übrigens auch die historische Ebene der Einzelsprachen von derjenigen der Diskurstraditionen zu unterscheiden erlaubt. Kontingent bedeutet ,nicht motiviert', d.h. die Präsenz und Ausprägung mancher sprachlicher Struktu- 
ren ist nicht unmittelbar funktional zu erklären, auch nicht aus der Kommunikationssituation heraus oder, das wurde in zahlreichen Lehrveranstaltungen Oesterreichers in München ganz deutlich, aus den Varietäten konstituierenden außersprachlichen Faktoren (Raum, Zeit, Gesellschaft, Situation). Wahrscheinlich ist hierunter auch ein Spezifikum der menschlichen Sprache zu fassen, nämlich die Eigenschaft der Wohlgeformtheit ohne jegliche kommunikative Fundierung, die eine verstehbare Äußerung ( ${ }^{\star}$ Morgen ich Tomaten kaufen wollen) von einer als zum eigenen Regel- und Normgefüge zugehörig erkennbaren (Ich will morgen Tomaten kaufen) abzugrenzen erlaubt (s. dazu ausführlich Seiler 2015, der wie Wulf Oesterreicher die Notwendigkeit eines funktionalen, aber auch autonomen Beschreibungs- und Erklärungszugriffs gerade auf grammatische Phänomene postuliert). Gut illustrieren lässt sich Kontingenz bzw. Wohlgeformtheit anhand der Syntax des deutschen Expletivums es oder auch anhand der Existenz einer syntaktischen Einheit Subjekt, die weder semantisch (Subjekte sind nicht zwingend Agentes) noch informationsstrukturell (Subjekte können Topics, Foki usw. sein) oder kommunikativ-diskursiv begründbar ist - Subjekte in Akkusativsprachen und ihre Eigenschaften sind kontingente, rein syntaktisch bedingte Erscheinungen (cf. Seiler 2015, 246f.).

Wie, wodurch und warum genau kontingente sprachliche Merkmale entstehen, sich verbreiten und vergehen, ist kaum Gegenstand des Oesterreicherschen Euvres - wohl aber ihre genaue vergleichende Beschreibung (cf. Oesterreicher 1992, 1996a, 1996b), die die Existenz der beschriebenen Phänomene wohltuend unverbunden zu vermeintlichen außersprachlichen Einflussfaktoren zur Kenntnis nimmt. Ob die Oesterreichersche Sprachwissenschaft damit in einen „sozialtheoretischen" (Selig 2017, 124) Rahmen zu stellen ist, vermag ich nicht zu beurteilen; ich würde annehmen, nicht mehr als die de Saussuresche. Und es stellt sich die Frage, ob zur Untersuchung der „Eigenlogik, die [Sprachwissen] als Wissen (und nicht Handeln) hat“ (Selig 2017, 128) die „Rückbindung des Sprachwissens an das soziale Handeln mitzudenken“ ausnahmslos notwendig ist oder nicht vielleicht gelegentlich sogar erkenntnisverhindernd sein kann - eine methodologische Frage, deren auch empirische Beantwortung den Rahmen dieses Beitrags sprengen würde ${ }^{5}$. Aber unabhängig vom Zugang - sozial- oder hand-

5 Wir würden eher davon abraten, bei sprachwissenschaftlichen Beschreibungen und Argumentationen ,immer den kommunikativen Gesamtzusammenhang mitzudenken“ (Selig 2017, 128, meine Hervorhebung); unten in Abschnitt 3 werden wir grammatische Variation thematisieren, deren kommunikative Einbettung - und dies auch nur mit Einschränkung - nur in Bezug auf die varietätenlinguistische Markierung der einzelnen Strukturen berücksichtigt werden kann; zum Verständnis der inneren Systematik der Kongruenzmuster in den einzelnen Konstruktionen trägt sie schlicht nichts bei. 
lungstheoretisch, semiotisch, deskriptiv-quantitativ, modular - der Kern sprachwissenschaftlicher Beschreibung und Reflexion bleiben im Oesterreicherschen Sprachdenken die sprachlichen Regel- und Normgefüge als solche.

Oesterreicher hält bei seinen Reflexionen um den Kern der Sprachwissenschaft wiederholt einen ganz wesentlichen Punkt fest (cf. Oesterreicher 2010b, 90, s. bereits Oesterreicher 2009, 102f.): Keine andere Disziplin als die Linguistik kann diese Regel- und Normgefüge als solche, als überindividuelle, nur vom konkreten Diskurs oder Text abstrahierend erfassbare und eben auch nicht unmittelbar kommunikativ bedingte, erforschen, erfassen und erklären, keine andere Disziplin strebt aber auch danach (cf. Oesterreicher 2009, 102f.; Oesterreicher 2010b, 90). Das Forschen nach der überindividuellen einzelsprachlichen historischen Regularität, nicht nach dem Individuell-Punktuellen, begründet meines Erachtens die von Wolf-Dieter Stempel bereits 1988 festgehaltene ,grundsätzliche Verschiedenheit von sprachwissenschaftlichem und literaturwissenschaftlichem Ansatz“ (Stempel 1988, 51), und der sprachwissenschaftliche ist durch seinen Gegenstand, die historischen Einzelsprachen und Varietäten als solche, eben auch grundsätzlich zu unterscheiden von einem kommunikations-, kultur- oder sozialwissenschaftlichen (so Oesterreicher 2009, 87). Noch früher, 1982 schon, haben das Theo Vennemann und Renate Bartsch folgendermaßen festgehalten:

Für die Linguistik ist Kerngebiet und Ausgangspunkt der Sprachtheorie die Theorie der Sprachsysteme, also gerade derjenige Ausschnitt der Sprachtheorie, der für die anderen Disziplinen nicht zentral ist, den sie vielmehr - bei genauerer Betrachtung - immer schon voraussetzen. Ähnliches gilt für die Theorie der Sprachveränderung, sofern sich nämlich die anderen Disziplinen überhaupt für sie interessieren. (Bartsch/Vennemann 1982, viii)

Anders gewendet: Wenn die Sprachwissenschaft in Forschung und Lehre diesen Kern der Linguistik verlässt, ihr „Herzstück“ nicht mehr wissenschaftlich betrachtet und reflektiert, dann tut es niemand (ich möchte hinzufügen: richtig). Die Gefahr einer gewollten oder unreflektierten metonymischen Verschiebung (Michele Loporcaro, p.c.) von der Untersuchung von Sprache (= Linguistik) in der Kommunikation bzw. Interaktion auf die Kommunikation bzw. Interaktion selbst bestand und besteht derzeit durchaus. Kommunikation kann auch ohne Sprache stattfinden und analysiert werden, fällt dann aber nicht mehr in den Untersuchungsbereich der Linguistik, die in einer solchen disziplinären Desorientierung eine Forschungslücke entstehen lässt, mit weitreichenden Konsequenzen bis in die Lehrerbildung hinein. Daher möchte ich eine Lanze für die sorgfältige disziplinäre Verortung der Linguistik brechen sowie für die klare Anerkennung ihres Kernbereichs, für die wichtige Beobachtung schließlich, dass dieser Kernbereich eigenständig ist und keine anderen Wissenschaften benötigt - was interdisziplinäre Fragestellungen und Forschungsprojekte etwa zwischen Literatur- 
wissenschaft und Linguistik gerade nicht ausschließt, sondern bei großer disziplinärer Sorgfalt überhaupt erst ermöglicht (so etwa Oesterreicher 2014, 302, s. auch Oesterreicher 2016, 40).

Es soll uns also, prinzipiell und auch im Folgenden, um den „Weinberg der Sprache“ und seine Pflege gehen, um die Einzelsprachen und die Varietäten, die sich ihnen zuordnen lassen.

\section{Die „Konstruktion von Varietäten“}

Als eine Aufgabe der Varietätenlinguistik benennt Oesterreicher in dem Text, auf den sich ein Teil des Titels meines Beitrags bezieht (Oesterreicher 2009, 112), die „Bestimmung der Varietäten einer historischen Einzelsprache“, die ohne die Reflexion und Miteinbeziehung der Diskurstraditionen nicht zu leisten sei. In Oesterreicher (2010a) im Titel sowie in Oesterreicher (2016) spricht Oesterreicher von der „Konstruktion von Varietäten“ (Oesterreicher 2016, 47-56), welche aus meiner Sicht und aus derjenigen von Andreas Dufter (s. dazu etwa Dufter/Stark 2002, womit Oesterreicher sich hier erstmals ausführlich auseinandersetzt) durchaus einen der problematischeren Aspekte des Koch/Oesterreicherschen Modells darstellt. Die Varietätenkonstruktion scheint nämlich quasi ,aus dem grammatischen Nichts' zu erfolgen (sie ist in der Tat handlungs- und ggf. sogar sozialtheoretisch determiniert, s. hierzu Selig 2017, 132-138), jedenfalls die Zuordnung bestimmter sprachlicher Erscheinungen $\mathrm{zu}$ einer bestimmten Dimension und einer bestimmten Varietät. Dies liest sich in der neuesten Publikation folgendermaßen:

\footnotetext{
Vor allem werden die im Französischen relativ zahlreichen historisch-einzelsprachlich ,disjunkten` Phänomene der Dimension 1b [= nähesprachliches Französisch, informell, ,gesprochen'], also etwa bei der Negation, dem passé simple, der Inversionsfrage, bei on statt nous und ça statt cela, beim accord des participe passé, bei Allomorphien der Personalklitika [...] usw. [...] kritisch besprochen. Bekanntlich können diese zwar historisch kontingenten, aber eben nicht diasystematisch markierten Phänomene, bei denen allein die Kennzeichnung ,gesprochen' vs. ,geschrieben ' trägt und die als ,hartes Faktum‘ das Französische vergleichsweise stark prägen, in dieser Sprache sogar als Beleg für eine diglossische Tendenz angeführt werden. (Oesterreicher 2016, 52f.)
}

Dieser Zuordnung der genannten sprachlichen Phänomene (Regeln oder Normen, cf. Koch 1999 dazu), jedenfalls Regularitäten, die ich nun im Einzelnen nicht weiter diskutieren oder illustrieren will mit einer Ausnahme, liegt im Koch/Oesterreicherschen Euvre, wenn ich recht sehe, die ursprüngliche empirische Arbeit aus den achtziger Jahren mit relativ kleinen und heutigen Standards 
wohl nicht mehr entsprechenden Korpora zugrunde, sowie eine Rezeption wichtiger Publikationen zum Thema, neben der grundlegenden von Ludwig Söll (31985) sicherlich auch derjenigen von Anne Zribi-Hertz (cf. Zribi-Hertz 2011) und Benjamin Massot (cf. Massot 2010) zur Diglossiefrage in einem eher formalen theoretischen Rahmen. D.h. die empirische Evidenz für diese Zuordnung ist nicht überwältigend groß (das gilt in vielen Bereichen der Varietätenlinguistik; cf. dazu neuerdings Krefeld 2016) und kann daher meines Erachtens durchaus problematisiert werden. Die Markiertheit einzelner Varianten als typisch dialektal, bildungsfern oder eben einfach ,informell', ,gesprochen' ist dabei, sehr wichtig, eine im engeren Sinne nicht-sprachliche (und meiner persönlichen Auffassung nach auch nicht unmittelbar sprachwissenschaftlich relevante) mentale Vorstellung der Sprecher, die als Teil ihres metasprachlichen, v.a. pragmatisch-kommunikativen und sozialen Wissens allerdings natürlich von Interesse ist.

Zugespitzt könnte man an diesem Punkt zusammenfassend festhalten, dass die „Konstruktion von Varietäten“, die den sozialen, auch sprachpolitischen Status von Idiomen oder die kommunikativen Gegebenheiten, immer wieder auch die technologischen Umwälzungen unserer Kommunikationsgewohnheiten in einzelnen Gesellschaften, miteinbeziehen muss, also Erkenntnisse aus Nachbardisziplinen wie der Soziologie oder der Kommunikationswissenschaft, im strengen Sinne nicht zu dem eingangs zitierten „Herzstück der Sprachwissenschaft“ gehört. Sie befasst sich nämlich mit der Klassifikation von Regel- und Normgefügen als dialektale, soziale, stilistische usw., d.h. mit der Zuordnung von Sprachlichem zu auch außersprachlich konstituierten kommunikativen Räumen im weitesten Sinne. In dieser Verschärfung würde dann einer der Kernbereiche des Oesterreicherschen Euvres nicht dem von ihm identifizierten Kernbereich der Sprachwissenschaft zugehören, oder in einer ,Meta-Relation“ dazu stehen - was so selbstverständlich zu kurz gegriffen ist. Die Aufgaben der Varietätenlinguistik gehen nämlich, dies wird in den neueren Publikationen allerdings nicht mehr explizit festgestellt, über die Bestimmung oder Konstruktion von Varietäten hinaus. Nach meiner Lesart des bisher Referierten zum „Weinberg der Sprache“ sollen in einem disziplinären Weinbau der Linguistik auch und vor allem die auch als „historisch kontingent“ eingestuften „Regel- und Normgefüge“ als solche beschrieben und idealiter erklärt werden, die wir in einzelnen Varietäten vorfinden, also schlicht, die sprachlichen Merkmale derselben. Beschreiben und erklären sollten wir sie als SprachwissenschaftlerInnen nach allem bisher Gesagten zuallererst anhand der der Sprache eigenen Regeln, was ich hier in einem ganz allgemeinen Sinne als Grammatik bezeichnen will. Wir können demnach, ausgehend von der großen klassifikatorischen Leistung von Peter Koch und Wulf Oesterreicher in Bezug auf das, was wir insbesondere im 
modernen Französischen Frankreichs und der europäischen Frankophonie (in Kanada sind die Verhältnisse teilweise anders; zur Negation s. etwa Poplack/StAmand 2009) vorfinden, ein ganzes Forschungsprogramm entwickeln, wenn wir nach dem Warum der Zuordnung einzelner der oben erwähnten Varianten zum „nähesprachlichen Französischen“ fragen - also eine Frage stellen, die wir außersprachlich-soziologisch (stigmatisiert, weil normfern), außersprachlich-psychologisch (ökonomischer aufgrund von Analogie und geringerer Komplexität, was immer man darunter verstehen mag), aber eben auch sprachwissenschaftlich beantworten können. Warum, das möchte ich exemplarisch im Folgenden fragen und versuchen $\mathrm{zu}$ beantworten, ist die Abwesenheit des accord du participe passé in Konstruktionen mit dem Hilfsverb avoir eine Variante des Nähefranzösischen, des „français de l'immédiat“, der „Dimension 1b“, und warum ist eine durchgehend korrekte Realisierung dieses accord ein Distanzsignal? Dies ist übrigens in den erwähnten Arbeiten zu einer möglichen Diglossie im Französischen so nicht festgestellt worden. Wichtiger aber: Die Varianten des Nähebereichs sind insgesamt, zumindest im Französischen, als Regel- und Normgefüge noch kaum verstanden worden, häufig auch gar nicht als solche in ihren grammatischen Eigenschaften untersucht - betreiben wir also gemeinsam ein wenig viticulture linguistique.

\subsection{Der accord du participe passé als Regel}

Unter (1) bis (3) finden sich im folgenden Beispiele zur Illustration der Regularitäten des accord du participe passé im modernen Standardfranzösischen:

(1) a. La

pomme $_{2}$ a été

ART.F.sg Apfel.F.sg hat.3sg gewesen

donné-e à $\mathrm{Jean}_{3}$.

,Der Apfel wurde Jean gegeben.

b. Pierre $_{1}$ a donné la pomme $_{2}$ à Jean .

Pierre hat.3sg gegeben ART.F.sG Apfel.F.sG an Jean

,Pierre hat Jean den Apfel gegeben.‘

(2) Marie $_{2}$ est arrivé-e.

Marie ist.3sg angekommen-F.sG

,Marie ist angekommen.

(3) a. Pierre ${ }_{1} \boldsymbol{l}_{2}{ }^{\prime}$ a donné-e à Jean $_{3}$.

Pierre PRO.AKк.F.SG hat.3sg gegeben-F.SG an Jean

,Pierre hat ihn (= den Apfel) Jean gegeben. 


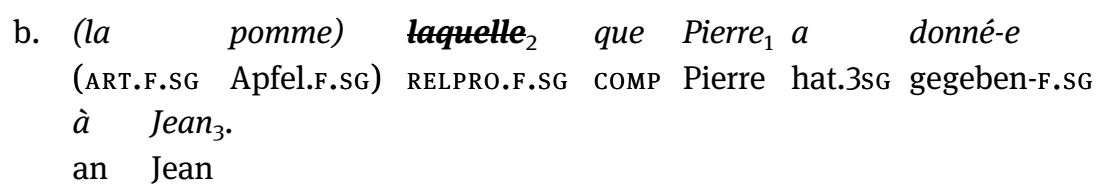
,(den Apfel), welchen Pierre Jean gegeben hat.‘

In Beispiel (1) sehen wir einen Passivsatz, das betroffene Objekt des Gegebenwerdens oder das interne Argument, also das nicht-agentivische zweite des trivalenten Verbs donner (daher die tiefgestellte 2), steht in Subjektposition, geht dem Partizip donné somit voran und löst Kongruenz aus (graphisch durch ein <-e> markiert). Dies erfolgt in (1b) nicht, da das Themaargument hier in seiner ursprünglichen postverbalen Position stehen bleibt. In (2) ist ein sogenanntes unakkusativisches Verb verwendet, dessen einziges Argument eher Objekteigenschaften hat (cf. Perlmutter 1978) und deshalb ebenfalls als internes Argument angesehen wird. Es steht hier in präverbaler Position, ganz vergleichbar dem Subjekt in (1a), und löst Kongruenz aus. In beiden Fällen der Beispiele unter (3) gehen pronominale Ersetzungen des Themaarguments dem Partizip linear voran - in (3a) als klitisches Pronomen, $l a$, in $3 \mathrm{~b}$ als Relativelement ${ }^{6}$ - und es erfolgt Kongruenz. Diese unterbleibt somit nur in (1b), wo das Themaargument dem Partizip folgt.

Der accord du participe passé existiert in verschiedenen Ausprägungen in allen romanischen Sprachen und Dialekten, wobei genau die auch varietätenlinguistisch interessante Regelkonstellation des Französischen - accord mit avoir ausgelöst durch ein pronominales dem Partizip vorangehendes Objekt wie in (3a) und (3b), zusammen mit weiteren Subregularitäten, auf die ich hier nicht weiter eingehen möchte (s. dazu Kayne 1989, 2015; Stark/Riedel 2012; auch Georgi 2014 in einer theoretischen Arbeit zu zahlreichen auch außereuropäischen Sprachen), so nur im Standardfranzösischen zu finden ist und dies wohl auch erst seit dem 17. Jahrhundert (cf. Levitt 1973, 26-28). Bemerkenswert - und eine erste Erklärungsmöglichkeit für ihre vermeintliche Abwesenheit im nähesprachlichen Französischen - ist dabei die Tatsache, dass spätestens seit dem 19. Jahrhundert (cf. Hunnius 1980) zumindest im Französischen Frankreichs die Partizipialkongruenz lautlich nicht mehr zu realisieren ist und entsprechend auch gar nicht zu hören -

6 In restriktiven Relativsätzen kann das genus- und numerusmarkierte lequel nicht in Objektposition stehen, sondern muss durch den unmarkierten Komplementierer que ersetzt werden (cf. Stark 2016a). Wir geben es in den Beispielen dennoch (durchgestrichen) an, um die Kongruenzmerkmale des Kongruenzauslösers - das vorangestellte Relativpronomen - in Erinnerung zu rufen. 
die meisten Partizipien enden auf Vokal, und bis auf einige Kantone der Westschweiz wird die Genusopposition heute nicht mehr realisiert. Ersetzen wir pomme durch lettre, donné durch écrit und arrivé durch mort in den bisher betrachteten Beispielen (cf. (4) bis (6)), wird der Unterschied in phonetischer Transkription sofort deutlich, es ist immer eine Kongruenz-Markierung des Genus des accord auslösenden Elements (dem „controller“ nach Corbett 2006):
a. $L a$
lettre $_{2} \quad a$
été
écrit-e [ekrit]
(à $\mathrm{Jean}_{3}$ ).
ART.F.SG Brief.F.sG hat.3sg gewesen
geschrieben-F.sG (an Jean)

,Der Brief wurde an Jean geschrieben.
b. Pierre $_{1} a$
écrit [ekri] la
lettre $_{2} \quad\left(\grave{a} \quad \mathrm{Jean}_{3}\right)$.
Pierre hat.3sg geschrieben ART.F.SG
Brief.F.sg (an Jean)
,Pierre hat Jean den Brief geschrieben.

(5)
Marie $_{2}$ est
mort-e [mort].
Marie ist.3sg
gestorben-F.sG
,Marie ist gestorben.

(6)
a. Pierre $_{1} l_{2}{ }^{\prime} \quad a \quad$ écrit-e [ekrit] (à Jean $\left.{ }_{3}\right)$.
Pierre PRo.AKк.F.sg hat.3sg geschrieben-F.sg (an Jean)
,Pierre hat ihn (= den Brief) an Jean geschrieben.
b. (la lettre) taquelle $_{2}$ que Pierre ${ }_{1}$ a
ART.F.SG Brief.F.SG RELPRO.F.SG COMP Pierre hat.3sg
écrit-e [ekrit] (à $\left.\mathrm{Jean}_{3}\right)$.
geschrieben-F.sG (an Jean)
,(den Brief), welchen Pierre Jean geschrieben hat.‘

Der Kongruenz auslösende Faktor scheint also die Reihenfolge zu sein: Kongruenz erfolgt, wenn der controller, das interne Argument des Verbs, welches dem Partizip zugrunde liegt, dem Partizip vorangeht, aus verschiedenen Gründen (in Subjektposition mit être, als Objektklitikon, als Reflexivum, wieder mit être, oder Relativelement). So ist die Regel auch in der berühmtesten und meistzitierten Modellierung formuliert worden, im Rahmen der generativen Grammatik nämlich, bei Richard Kayne (1989), und sie war und ist dort Grundlage für Theoretisierungen über Kongruenz überhaupt, die nach Kayne immer nur in einer spezifischen syntaktischen Konfiguration (zwischen einem Kopf und seinem Spezifizierer) erfolgen kann und daher mit verschiedenen Prozessen der syntaktischen Bewegung, also Reihenfolgeänderungen, einhergeht (s. kürzlich die Dissertation von Doreen Georgi aus Leipzig 2014). Nur: Dies entspricht nicht den französischen Tatsachen (Beispiele (7) und (8)): 
(7) a. Une épreuve sera présenté-e à chaque candidat. ART.F.SG Test.F.SG sein.Fut.3sg vorgelegt-F.SG an jeder Kandidat ,Ein Test wird jedem Kandidaten vorgelegt.

b. A chaque candidat sera présenté-e une épreuve. An jeder Kandidat sein.FUT.3sg vorgelegt-F.sg ART.F.sg Test.F.sG ,Jedem Kandidaten wird ein Test vorgelegt.

(8) a. Je voudrais que soient inscrit-s tous Ich wollen.cond.1sG comp sein.sBJv.3PL eingeschrieben-PL alle.PL les enfants de Marie.

ART.PL Kinder.M.PL von Marie

,Ich hätte gern, dass alle Kinder von Marie eingeschrieben sind. ‘

b. ${ }^{\star}$ Je voudrais que soient tous inscrit-s

Ich wollen.cond.1sg comp sein.sBJv.3PL alle.PL eingeschrieben-PL les enfants de Marie.

ART.PL Kinder.M.PL von Marie

^,Ich hätte gern, dass die Kinder eingeschrieben sind alle von Marie.‘

Karen Lahousse hat nämlich in einer herausragenden Monographie über verschiedene Typen von Verb-Subjekt-Inversion im Gegenwartsfranzösischen u.a. gezeigt, dass Partizipialkongruenz auch möglich ist etwa mit Subjekten von Passivkonstruktionen, die als zugrundeliegende Themata im Sinne von, semantischen Objekten' in ihrer postverbalen Position verbleiben und dennoch accord auslösen, wie in den Beispielen (7) und (8) illustriert (Lahousse 2011, Kapitel 3.3). In (7b) befindet sich das interne Argument als Subjekt, une épreuve, am Satzende, und dennoch erfolgt Kongruenz auf dem Partizip présenté-e. Dass das interne Argument, tous les enfants de Marie, in seiner ursprünglichen Position verblieben ist in solchen Konstruktionen, zeigt u.a. (8b): Hätte es sich in eine Subjektposition bewegt, wäre sogenanntes Quantorenfloating möglich (les enfants sont tous inscrits, ,die Kinder sind alle eingeschrieben'), was bei internen Argumenten in postverbaler Position nicht geht. Das bedeutet, dass für den accord in Konstruktionen mit dem Hilfsverb être (,sein') die Reihenfolge zwischen Argument und Partizip prinzipiell keine Rolle spielt, für den accord mit dem Hilfsverb avoir (s. die Beispiele (1b) und (4b) vs. (3) und (6)) aber sehr wohl. Die Regel, die in (3b) bzw. (6b) illustriert ist, also accord mit einem vorangehenden Relativelement, scheint dabei besonders fehleranfällig zu sein - und sie ist in der Diachronie des Französischen ab dem 13. Jahrhundert bis zur Normierung im 17. Jahrhundert kaum mehr belegt (Brunot 1899, 523f.; Jensen 1990, 336) und auch in typologischer Hinsicht sehr markiert bzw. möglicherweise einzigartig, zumindest im Konzert der romanischen Gegenwartssprachen und -dialekte (wie Michele Loporcaro genau darlegt, s. Loporcaro 1998, 2010). 


\subsection{Der accord du participe passé in Korpora des Gegenwartsfranzösischen}

Sehen wir uns also an, was die Sprecher machen, auch unter Einbeziehung neuerer graphischer Daten, die von Wulf Oesterreicher als äußerst relevant bezeichnet wurden für die Varietätenlinguistik, nämlich Daten alltagssprachlicher, „pragmatischer Schriftlichkeit und die Reflexe sprechnaher Varietäten im graphischen Medium“ (Oesterreicher 2009, 113). Dabei nehmen wir, wie alle strukturalistischen Beschreibungsmodelle menschlicher Sprache, selbstverständlich an, dass die Daten des Sprachgebrauchs nicht mit dem zu rekonstruierenden Wissenssystem identisch sind (im Gegensatz zu sogenannten usage-based approaches neueren Datums), d.h. wir folgen de Saussure, Noam Chomsky und auch wiederum Wulf Oesterreicher in ihrer prinzipiellen (wiewohl natürlich in einzelnen Aspekten nicht identischen) methodologischen Trennung von Gebrauch und Sprachwissen. Alles Folgende muss also relativiert werden als individuell und punktuell; es kann niemals ein Beweis, wohl aber ein Indiz sein für die Natur der Wissensbestände, aufgrund derer die Sprecher operieren.

In den vier folgenden Tabellen finden sich Zahlen zur Realisierung des accord in vier verschiedenen Korpora des Gegenwartsfranzösischen, wobei für die drei phonischen nur die Fälle berücksichtigt wurden, wo tatsächlich regelkonform ein hörbarer accord zu erwarten wäre:

Tab. 1: Markierung des accord du participe passé in sms4science.ch

\begin{tabular}{llr}
\hline & Absolute Zahlen & Prozent \\
\hline Nicht markiert & 72 & 10.1 \\
\hline Markiert & 638 & $\mathbf{8 9 . 9}$ \\
\hline total & 710 & 100 \\
\hline
\end{tabular}

Tab. 2: Markierung des accord du participe passé in OFROM (Westschweiz)

\begin{tabular}{llr}
\hline & Absolute Zahlen & Prozent \\
\hline Nicht markiert & 14 & 14.43 \\
\hline Markiert & 83 & $\mathbf{8 5 . 5 7}$ \\
\hline Total & 97 & 100 \\
\hline
\end{tabular}


Tab. 3: Markierung des accord du participe passé im frz. Teil des C-ORAL-ROM

\begin{tabular}{llr}
\hline & Absolute Zahlen & Prozent \\
\hline Nicht markiert & 16 & 14.55 \\
\hline Markiert & 94 & $\mathbf{8 5 . 4 5}$ \\
\hline Total & 110 & 100 \\
\hline
\end{tabular}

Tab. 4: Markierung des accord du participe passé im frz. und schweizerischen Teil des PFC

\begin{tabular}{llr}
\hline & Absolute Zahlen & Prozent \\
\hline Nicht markiert & $7(\mathrm{~F})+1(\mathrm{CH})$ & $13.46(\mathrm{~F})-9.1(\mathrm{CH})$ \\
\hline Markiert & $45(\mathrm{~F})+10(\mathrm{CH})$ & $\mathbf{8 6 . 5 4 ( \mathrm { F } ) - \mathbf { 9 0 . 9 } ( \mathrm { CH } )}$ \\
\hline Total & $52(\mathrm{~F})+11(\mathrm{CH})$ & 100 \\
\hline
\end{tabular}

Wenn wir nur die fettgedruckten Zahlen in den vier Tabellen betrachten (cf. Stark 2015), sehen wir auf den ersten Blick Erstaunliches: In Westschweizer SMS (Tabelle 1), im gesprochenen Französisch der Westschweiz (Tabelle 2, das Korpus OFROM enthält auch Distanzsprachliches, aber eher wenig), im gesprochenen Französisch Frankreichs (Tabelle 3, verschiedene Kommunikationssituationen) und im spontansprachlichen Teil des PFC, einem gesprochenen Korpus aus allen Teilen der Frankophonie (Tabelle 4, Daten für Frankreich und die Westschweiz) realisieren die Sprecher bzw. Schreiber den accord du participe passé in 85-90 \% aller Fälle korrekt. D.h. dieser accord wird, unabhängig von der Kommunikationssituation bzw. in sogar eher nähesprachlichen Korpora, durchaus realisiert. Handelt es sich dabei nur um Fälle des accord mit être? Oder haben Koch/ Oesterreicher ([1990] 22011, 69) und Oesterreicher (2016, 52f.) schlicht unrecht? Oder haben wir es schließlich mit der von Oesterreicher angeführten „Freiheit des Sprechers“ (nach Herbert Pilch) zu tun?

Sprecher haben immer die Möglichkeit, in ihren Diskursen und Texten von den Linguisten konstatierte Regularitäten zu überspielen und zu ignorieren. (Oesterreicher 2016, 52)

Wie plausibel ist es aber, anzunehmen, dass die 85-90 \% korrekt markierter Fälle von Partizipialkongruenz in vier verschiedenen Korpora nur auf être fallen oder aber die Variation um avoir absichtlich konterkariert wird?

Folgen wir, um diese Fragen anzugehen, wieder Wulf Oesterreicher, der wiederholt auf den Unterschied zwischen sprachlichen Daten, wie sie uns bis jetzt in den vier Korpora als reine Zahlenverhältnisse des individuellen Gebrauchs 
entgegentreten, und linguistischen Fakten, die allein relevant sind für die sprachwissenschaftliche Modellierung, hingewiesen hat (zuletzt in Oesterreicher 2016, 55, s. v.a. Oesterreicher 2010). Wie werden aus sprachlichen Daten linguistische Fakten? Durch, wenn wir richtig lesen, „sprachwissenschaftliche Arbeit und Modellierung“ (Oesterreicher 2016, 55). Letztere, die natürlich auch und vor allem sprachwissenschaftliche Arbeit ist, ist in der Tat dringend geboten, würde den Rahmen dieses Beitrags aber sprengen und ist im formalsyntaktischen Sinne nicht ohne weiteres möglich (s. dazu Georgi/Stark in Vorbereitung): Ein Teil der Regeln, genauer diejenigen, die in (3b) und (6b) illustriert sind und die vermeintlich der nähe-distanzsprachlichen Opposition in der Realisierung des accord mit avoir zugrunde liegen, sind in keinem aktuellen generativen Modell von Kongruenz ohne Zusatzannahmen modellierbar (Chomsky 2001 et passim; D'Alessandro/Roberts 2008, 2010; Georgi 2014; Stark 2016b) - was ein wertvolles Indiz ist für das korrekte Verständnis unserer Varianten und ihrer varietätenlinguistischen Markierung. Ich möchte mich hier auf deskriptive sprachwissenschaftliche Arbeit im eingangs skizzierten Sinne beschränken: Betrachten wir die Regel- und Normgefüge des accord du participe passé in den französischen Daten als solche und untersuchen wir sie innersprachlich, nach ihrer Grammatik.

Ganz unabhängig von Fragen der varietätenlinguistischen Markierung bin ich auf der Suche nach dem innersprachlichen Faktor, der ein Ausbleiben der Kongruenz mit statistischer Signifikanz in Westschweizer SMS auslöst, schon 2012 fündig geworden (cf. Stark/Riedel 2012). Andere Faktoren habe ich ebenfalls untersucht (s. dazu Stark/Riedel 2012), aber es ist ein einziger Faktor, der klar verantwortlich zu sein scheint, und er manifestiert sich auch klar in den gesprochenen Daten: Es ist die Konstruktion, wie man den folgenden vier Tabellen entnehmen kann:

Tab. 5: Markierung des accord du participe passé nach Hilfsverb in sms4science.ch

\begin{tabular}{lrrr}
\hline & Nicht markiert & Markiert & Total \\
\hline Avoir & 25 & 82 & 107 \\
& $\mathbf{2 3 . 4 \%}$ & $76.6 \%$ & $100 \%$ \\
\hline Etre & 32 & 377 & 409 \\
& $7.8 \%$ & $92.2 \%$ & $100 \%$ \\
\hline Etre Ellipsen/Epithet ${ }^{7}$ & 15 & 179 & 194 \\
& $7.7 \%$ & $92.3 \%$ & $100 \%$ \\
\hline Total & 72 & 638 & 710 \\
& $10.1 \%$ & $89.9 \%$ & $100 \%$ \\
\hline
\end{tabular}

7 Diese Kategorie umfasst Fälle von Auxiliarellipse wie in: (Je suis) désolée d'être en retard. 
Tab. 6: Markierung des accord du participe passé nach Hilfsverb in OFROM

\begin{tabular}{lrrr}
\hline & Nicht markiert & Markiert & Total \\
\hline Avoir & 7 & 8 & 15 \\
& $46.67 \%$ & $53.33 \%$ & $100 \%$ \\
\hline Etre & 7 & 55 & 62 \\
& $11.29 \%$ & $88.7 \%$ & $100 \%$ \\
\hline Etre Ellipsen/Epithet & 0 & 20 & 20 \\
& $0 \%$ & $100 \%$ & $100 \%$ \\
\hline Total & 14 & 82 & 97 \\
& $14.58 \%$ & $85.42 \%$ & $100 \%$ \\
\hline
\end{tabular}

Tab. 7: Markierung des accord du participe passé nach Hilfsverb im frz. Teil des C-ORAL-ROM

\begin{tabular}{lrrr}
\hline & Nicht markiert & Markiert & Total \\
\hline Avoir & 9 & 11 & 20 \\
& $45.0 \%$ & $55.0 \%$ & $100 \%$ \\
\hline Etre & 7 & 59 & 66 \\
& $10.6 \%$ & $89.39 \%$ & $100 \%$ \\
\hline Etre Ellipsen/Epithet & 0 & 24 & 24 \\
& $0 \%$ & $100 \%$ & $100 \%$ \\
\hline Total & 16 & 94 & 110 \\
& $14.55 \%$ & $85.45 \%$ & $100 \%$ \\
\hline
\end{tabular}

Tab. 8: Markierung des accord du participe passé nach Hilfsverb im frz. und schweizerischen Teil des PFC

\begin{tabular}{llll}
\hline & Nicht markiert & Markiert & Total \\
\hline Avoir & $\mathbf{5 ( F ) + 1 ( C H )}$ & $9(\mathrm{~F})+2(\mathrm{CH})$ & $14(\mathrm{~F})+3(\mathrm{CH})$ \\
& $\mathbf{3 5 . 7 1} \%-\mathbf{3 3 . 3} \%$ & $64.29 \%-66.7 \%$ & $100 \%$ \\
\hline Etre & $2(\mathrm{~F})+0(\mathrm{CH})$ & $23(\mathrm{~F})+6(\mathrm{CH})$ & $25(\mathrm{~F})+6(\mathrm{CH})$ \\
& $8 \%-0 \%$ & $92 \%-100 \%$ & $100 \%$ \\
\hline Etre Ellipsen/Epithet & $0(\mathrm{~F})+0(\mathrm{CH})$ & $13(\mathrm{~F})+2(\mathrm{CH})$ & $13(\mathrm{~F})+2(\mathrm{CH})$ \\
& $0 \%$ & $100 \%$ & $100 \%$ \\
\hline Total & $7(\mathrm{~F})+1(\mathrm{CH})$ & $45(\mathrm{~F})+10(\mathrm{CH})$ & $52(\mathrm{~F})+11(\mathrm{CH})$ \\
& $13.46 \%-9.1 \%$ & $86.54 \%-90.9 \%$ & $100 \%$ \\
\hline
\end{tabular}

In Fettdruck sehen wir die Fehlerraten für den accord du participe passé mit dem Hilfsverb avoir, d.h. in der Anwendung einer Regel, die auf der rein 
linearen Abfolge von Objektpronomen oder Relativpronomen und Partizip basiert (für être gilt dies ja nicht, s. die obigen Beispiele (7) und (8) und meine Kommentare dazu). Es ist diese Regel, die nicht beherrscht wird, und es ist dies ja genau auch die Variable, die für die Opposition Standard vs. „français de l'immédiat" interpretierbar ist. Obwohl die Fallzahlen jeweils klein sind, scheint es insbesondere im OFROM (Tabelle 6) und im C-ORAL-ROM (Tabelle 7) fast Zufall zu sein, ob die Kongruenz hier markiert wird oder nicht. Da Gleiches für die Konstruktionen mit être, dt. sein, nicht zutrifft, scheinen wir, mit den Mitteln der Korpuslinguistik und ohne weitere Vorannahmen, den ,Schuldigen' gefunden zu haben. Über die Regionen, Schichten und Stile hinweg, die in den vier Korpora abgebildet sind, wird die im innerromanischen Vergleich so nicht existierende reihenfolgebasierte Regel rund um das Hilfsverb avoir nicht konsequent angewendet. Sie ist diachron schon im Altfranzösischen kaum nachweisbar und bekanntermaßen erst im 16. Jahrhundert vom Dichter Clément Marot explizit formuliert.

Auf der Suche nach Erklärungsmöglichkeiten dieses Ergebnisses, das mit der Feststellung der nähesprachlichen Markierung eines ausbleibenden accord mit avoir absolut kompatibel ist, aber es eben als ein Explanandum betrachtet, haben wir erwähnt, dass die Einbeziehung von Forschungsergebnissen aus Typologie und formaler Linguistik die Einmaligkeit der standardfranzösischen Partizipialkongruenz mit avoir unter den Sprachen der Welt und in der Romania festzustellen und somit mindestens auf ihre starke Markiertheit zu schließen erlaubt. D.h. wir müssen im ,Weinberg der Sprache und ihrer Varietäten' stets disziplinär stringent, aber auch informiert argumentieren, nicht partialisiert (also nur innerhalb der romanistischen oder gar nur französischen Linguistik, oder nur innerhalb eines theoretischen Ansatzes oder Modells) und auch nicht unbedingt interdisziplinär (z.B. historisch oder soziologisch; so verstehe ich das Oesterreichersche Plädoyer am Ende von Oesterreicher 2015, 32-34). Dann sehen wir, dass die fragliche Kongruenz mit avoir keinerlei grammatische Funktion zu haben scheint, im Unterschied zu Konstruktionen mit être, in denen der accord meist markiert wird ${ }^{8}$. In solchen Konstruktionen ist nämlich das Subjekt nicht zugleich der Handelnde, das Agens, sie sind in engerem Sinne intransitiv, was durch den accord markiert werden kann (ähnliche Überlegungen zu einem funktionalen Wert des accord haben auch Kayne 1989 oder Loporcaro 1998 bereits formuliert).

8 Tatsächlich finden sich hier durchaus auch Nicht-Markierungen in den Korpusdaten, die sich aber noch in einem Toleranzbereich des Sprachgebrauchs bewegen („Performanz“). Die Signifikanz der Konstruktion (avoir - être) als mit der Realisierungsrate direkt korrelierter Faktor wird davon nicht beeinträchtigt. 
Bei Konstruktionen mit avoir, man vergleiche nur Beispiel (1b) mit den Beispielen in (3), ist das Subjekt aber agentiv - und dennoch muss manchmal der accord gemacht werden, manchmal nicht - er hat also nichts mit dem Konstruktionstyp, der Verteilung semantischer Rollen auf syntaktische Funktionen zu tun, mit dem Unterschied zwischen Transitivität und Intransitivität. Er ist eine servitude grammaticale, funktionslos und hochkomplex (weil reihenfolgebasiert) - und schwer zu erlernen, wie die psycholinguistische Forschung zum Französischen nachgewiesen hat (cf. Brissaud/Cogis 2008).

Und nun sind wir des Rätsels Lösung ganz nahe: Wenn die Behauptung, die Abwesenheit des accord du participe passé in Konstruktionen mit avoir im Französischen sei ein Merkmal des nähesprachlichen Französischen, auf der Produktionsseite empirisch nicht zu halten ist (s. die Zahlen in den Tabellen (5) bis (8)), so ist ihr Gegenstück durchweg korrekt: Die konsequente Markierung des accord du participe passé auch in den Fällen, die der natürlichen Syntax menschlicher Sprachen widersprechen, funktionslos und stark fehleranfällig sind, schließlich schwer und spät erlernt werden in der Schule, ist ein Merkmal des Standards, der kommunikativen Distanz, und nur des Standards, der im Französischen mehr als in anderen europäischen Nationalsprachen einen ganz besonders künstlichen, ja unnatürlichen Charakter hat aufgrund der externen Sprachgeschichte mit der starken Normierung seit dem 17. Jahrhundert und der Normfixiertheit der französischen Gesellschaft. Diese Normfixiertheit bewirkt auf der Perzeptionsseite möglicherweise die Etikettierung nicht markierter accords als nähesprachlich, informell, was allerdings noch empirisch zu überprüfen wäre (eventuell sind solche fehlende accord-Fälle durchaus diastratisch, also sozial niedrig, oder diaphasisch, stilistisch niedrig markiert); dazu kann man auf das schöne Diktum der „idéologie du standard“ von Milroy/Milroy (1985) verweisen. Hierfür hilft eine soziologische Einbettung und eine sozialtheoretische Analyse sprachlichen Handelns - das Warum des ausbleibenden accord mit avoir in nicht-normorientierten Kontexten im Unterschied zu être vermag sie niemals zu ergründen.

\section{Zusammenfassung und Ausblick}

Ausgehend von dem gewählten Einzelbeispiel, dem accord du participe passé im Französischen, ist es vermutlich insgesamt möglich, die oben zitierte Aufzählung v.a. morphosyntaktischer Phänomene als Merkmale einer konstruierten Varietät in der Dimension 1b, also des nähesprachlichen Französischen in der Nachfolge Ludwig Sölls, quasi als Negativbild der sehr heterogenen französischen Standardvarietät $\mathrm{zu}$ interpretieren, qui ne fait pas système im Sinne einer 
natürlichen menschlichen Einzelsprache (cf. Gadet 2007, 28) - genauso wenig allerdings wie die als „français de l'immédiat“ zu einer Varietät zusammengepackten Dinge. Dabei sind ihre bloße Existenz und v.a. ihre relativ hohe Frequenz ein Explanandum - ihre Stigmatisierung aus normativer Sicht als ,nähesprachlich', wenn wir Koch/Oesterreicher in Bezug auf das Französische so lesen wollen, kann auch sprachsoziologisch und historisch erklärt werden, dazu müssen wir den Kernbereich der Sprachwissenschaft noch nicht einmal betreten. Existenz, zugrundeliegende Regularitäten und Frequenz bleiben damit aber unerklärt, unerforscht. Warum, so die generelle Frage, werden bestimmte v.a. morphosyntaktische Phänomene des Standards nicht beherrscht, und zwar so häufig und von derart verschiedenen Sprechergruppen, dass diese Abweichungen nur noch ,nähesprachlich' sind für die Hörenden? Um zu einer erklärenden Analyse des Phänomens dieser Varietäten oder allgemeiner und vorsichtiger der beobachteten Variation im modernen Französischen zu gelangen, kommen wir um den von Wulf Oesterreicher seit seinen frühesten Arbeiten klarsichtig identifizierten Kernbereich der Sprachwissenschaft, also die Untersuchung der fraglichen Regel- und Normgefüge als solche, nicht herum - wir müssen uns die Satzkonstruktionen, die Bedeutungen, die morphologischen Elemente in ihrer Distribution und phonologischen Realisierung ansehen, wir müssen Grammatikforschung betreiben. Und dies möglichst vor dem Hintergrund eines Grammatikmodells, denn auch die Modellierung sprachlicher Daten, um diese zu Fakten werden zu lassen, wird von Oesterreicher gefordert im Kernbereich der Sprachwissenschaft - welchen Modells, mag hier offenbleiben. Es sollte meiner Ansicht nach erklärungsmächtig genug sein, um unabhängig vom Betrachter die beobachteten Daten zu linguistischen Fakten werden zu lassen und nicht einfach nur in anderer Form wieder aufzuzählen oder gar subjektiv zu interpretieren. Es sollte wenn möglich die verschiedensten Beschreibungsebenen menschlicher Sprache sowohl getrennt als auch in ihrem Zusammenwirken beschreiben und erklären können und dabei genuin sprachliche (ich erinnere an das Faktum der Wohlgeformtheit) von allgemein-kognitiven und -kommunikativen Mechanismen unterscheiden helfen. Diese Grammatikforschung steht und stand nie im Gegensatz, sondern immer im Dienste der Erforschung und Erklärung sprachlicher Variation.

Wir stehen somit vor einer gewaltigen Aufgabe - Wulf Oesterreichers sprachtheoretische Arbeit und seine ganz konkreten Aussagen zu verschiedenen varietätenlinguistischen Phänomenen als Forschungsprogramm ernst zu nehmen, kritisch $\mathrm{zu}$ diskutieren, empirisch $\mathrm{zu}$ untermauern - oder auch $\mathrm{zu}$ widerlegen, auch das ist wissenschaftlicher Fortschritt. Wir sind in einer komfortablen Situation, denn wir können ausgehen von einer Vielzahl von oft schnell skizzierten durchaus richtigen Beobachtungen und Überlegungen, die es nun gilt, genauer 
zu untersuchen und zu verstehen. Dies sollten wir tun in Kenntnis ihrer Spezifik als romanische Daten und Fakten, aber niemals isoliert vom Erkenntnisfortschritt unserer Disziplin, der Sprachwissenschaft.

\section{Bibliographie}

\section{Corpora}

C-ORAL-ROM: Cresti, Emanuela/Moneglia, Massimo (2005): C-ORAL-ROM. Integrated Reference Corpora for Spoken Romance Languages, Amsterdam, Benjamins.

OFROM: Corpus oral de français parlé en Suisse Romande. http://www11.unine.ch.

(Cf. Avanzi, Matthieu/Béguelin, Marie-José/Diémoz, Federica (2012-2014): „Présentation du corpus OFROM - corpus oral de français de Suisse romande“, Université de Neuchâtel. http://www.unine.ch/ofrom).

PFC: Phonologie du français contemporain. http://www.projet-pfc.net.

(Cf. Durand, Jacques/Laks, Bernard/Lyche, Chantal (2002): „La phonologie du français contemporain: usages, variétés et structure“, in: Claus Pusch/Wolfgang Raible (Hg.), Romanistische Korpuslinguistik - Korpora und gesprochene Sprache/Romance Corpus Linguistics Corpora and Spoken Language, Tübingen, Narr, 93-106.)

www.sms4science.ch: Stark, Elisabeth/Ruef, Beni/Ueberwasser, Simone (2009-2014): Swiss SMS Corpus, University of Zurich. https://sms.linguistik.uzh.ch.

\section{Forschungsliteratur}

Bartsch, Renate/Vennemann, Theo (1982): Grundzüge der Sprachtheorie. Eine linguistische Einführung, Tübingen, Niemeyer.

Brissaud, Catherine/Cogis, Danièle (2008): „L'accord du participe passé. Reconsidération d'un problème ancien à la lumière de données récentes sur l'acquisition“, in: Jacques Durand/ Benoît Habert/Bernard Laks (Hg.), Congrès mondial de linguistique française, Paris, 9-12 juillet 2008, 413-424.

Brunot, Ferdinand (1899): Précis de grammaire historique de la langue française, Paris, Masson et Cie.

Chomsky, Noam (2001): „Derivation by Phase“, in: Michael Kenstowicz (Hg.), Ken Hale. A Life in Language, Cambridge (Mass.), MIT Press, 1-52.

Chomsky, Noam (2005): „Three Factors in Language Design“, in: Linguistic Inquiry 36/1, $1-22$.

Chomsky, Noam (2007): „Approaching UG from below“, in: Uli Sauerland/Hans-Martin Gärtner (Hg.), Interfaces + recursion = language?, New York, Mouton de Gruyter, 1-29.

Chomsky, Noam (2008): „On phases“, in: Robert Freidin/Carlos Potero/Maria Luisa Zubizarreta (Hg.), Foundational issues in linguistic theory: essays in honor of Jean-Roger Vergnaud, Cambridge (Mass.), MIT Press, 133-166.

Corbett, Greville G. (2006): Agreement, Cambridge, Cambridge University Press. 
Coseriu, Eugenio (1974): Synchronie, Diachronie und Geschichte. Das Problem des Sprachwandels, München, Fink.

Coseriu, Eugenio ('1981): Textlinguistik. Eine Einführung, Tübingen, Narr.

Coseriu, Eugenio (1988): Einführung in die allgemeine Sprachwissenschaft, Tübingen, Francke.

D’Alessandro, Roberta/Roberts, Ian (2008): „Movement and Agreement in Italian Past Participles and Defective Phases“, in: Linguistic Inquiry 39/3, 477-491.

D’Alessandro, Roberta/Roberts, lan (2010): „Past participle agreement in Abruzzese: split auxiliary selection and the null-subject parameter“, in: Natural Language and Linguistic Theory $28,41-72$.

Dufter, Andreas/Stark, Elisabeth (2002): „La variété des variétés. Combien de dimensions pour la description? Quelques réflexions à partir du français“, in: Romanistisches Jahrbuch 53, 81-108.

Feilke, Helmuth/Hennig, Mathilde (Hg.) (2016): Zur Karriere von ,Nähe und Distanz'. Rezeption und Diskussion des Koch-Oesterreicher-Modells, Berlin/New York, de Gruyter.

Gadet, Françoise (2007): La variation sociale en français, Gap, Ophrys.

Georgi, Doreen (2014): Opaque Interactions of Merge and Agree: On the Nature and Order of Elementary Operations, unpublished PhD thesis, Universität Leipzig.

Georgi, Doreen/Stark, Elisabeth (in Vorbereitung): „Past participle agreement in French - one or two rules?“, in: Marc-Olivier Hinzelin/Natascha Pomino/Eva-Maria Remberger (Hg.), Selected Papers from the workshop „The Morphosyntax of Romance languages and its Formal Analysis“. 35. Deutscher Romanistentag 2017, Zürich.

Haegeman, Liliane (1997): „Register variation, truncation and subject omission in English and in French“, in: English Language and Linguistics 1, 233-270.

Haegeman, Liliane (2013): „The syntax of registers. Diary subject omission and the privilege of the root", in: Lingua 130, 88-110.

Hauser, Marc/Chomsky, Noam/Fitch, Tecumseh (2002): „The Faculty of Language: What Is It, Who Has It, and How Did It Evolve?“, in: Science 298, 1569-1579.

Hunnius, Klaus (1980): „Sprachnorm und Sprachgebrauch: Zur Geschichte der Kongruenzregeln des participe passé“, in: Hans-Dieter Bork/Artur Greive/Dieter Woll (Hg.), Romanica Europaea et Americana. Festschrift für Harri Meier zum 75. Geburtstag, Bonn, Bouvier, 131-169.

Jensen, Frede (1990): Old French and Comparative Gallo-Romance Syntax, Tübingen, Niemeyer.

Kayne, Richard (1989): „Facets of Romance past participle agreement“, in: Paola Benincà (Hg.), Dialect variation and the theory of grammar, Dordrecht, Foris, 85-103.

Kayne, Richard (2015): „Two Notes on Deletion“. Vortrag am 41st Incontro di Grammatica Generativa, Perugia, Italien, Februar 28, 2015.

Koch, Peter (1999): „Gesprochen/geschrieben - eine eigene Varietätendimension?“, in: Norbert Greiner/Joachim Kornelius/Giovanni Rovere (Hg.), Texte und Kontexte in Sprachen und Kulturen. Festschrift für Jörn Albrecht, Trier, Wissenschaftlicher Verlag Trier, 141-168.

Koch, Peter/Oesterreicher, Wulf ([1990 $\left.{ }^{2} 2011\right)$ : Gesprochene Sprache in der Romania. Französisch - Italienisch - Spanisch, Berlin, de Gruyter.

Krefeld, Thomas (2016): „Profilo sociolinguistico“, in: Sergio Lubello (Hg.), Manuale di linguistica italiana, Berlin/New York, de Gruyter, 262-274.

Lahousse, Karen (2011): Quand passent les cigognes? Le sujet nominal postverbal en français moderne, Paris, Presses Universitaires de Vincennes.

Levitt, Jesse (1973): „The Agreement of the Past Participle in Modern French: Orthographic Convention or Linguistic Fact?“, in: Linguistics 114, 25-41. 
Loporcaro, Michele (1998): Sintassi comparata dell'accordo participiale romanzo, Turin, Rosenberg e Sellier.

Loporcaro, Michele (2010): „Variation and change in morphology and syntax. Romance object agreement“, in: Franz Rainer/Wolfgang U. Dressler/Dieter Kastovsky/Hans Christian Luschützky (Hg.), Variation and Change in Morphology. Selected Papers from the 13th international morphology meeting, Vienna, February 2008, Amsterdam/Philadelphia, Benjamins, 149-175.

Massot, Benjamin (2010): „Le patron diglossique de variation grammaticale en français“, in: Langue Française 168, 87-106.

Milroy, James/Milroy, Lesley (1985): Authority in language: investigating language prescription and standardisation, London, Routledge \& Paul.

Oesterreicher, Wulf (1979): Sprachtheorie und Theorie der Sprachwissenschaft, Heidelberg, Winter.

Oesterreicher, Wulf (1992): „SE im Spanischen. Pseudoreflexivität, Diathese und Prototypikalität von semantischen Rollen“, in: Romanistisches Jahrbuch 43, 237-260.

Oesterreicher, Wulf (1996a): „Gemeinromanische Tendenzen V: Morphosyntax“, in: Günter Holtus/Michael Metzeltin/Christian Schmitt (Hg.), Lexikon der Romanistischen Linguistik, Bd. II.1, Tübingen, Niemeyer, 273-309.

Oesterreicher, Wulf (1996b): „Gemeinromanische Tendenzen VI: Syntax“, in: Günter Holtus/ Michael Metzeltin/Christian Schmitt (Hg.), Lexikon der Romanistischen Linguistik, Bd. II.1, Tübingen, Niemeyer, 309-355.

Oesterreicher, Wulf (2009): „Der Weinberg des Textes: Die Philologien und das Projekt, Textwissenschaft" im Horizont einer sozialen Semiotik“, in: Germanisch-romanische Monatsschrift 59, 81-118.

Oesterreicher, Wulf (2010a): „Sprachliche Daten und linguistische Fakten - Variation und Varietäten. Bemerkungen zu Status und Konstruktion von Varietäten, Varietätenräumen und Varietätendimensionen“, in: Vilmos Ágel/Mathilde Hennig (Hg.), Nähe und Distanz im Kontext variationslinguistischer Forschung, Berlin/New York, de Gruyter, 23-62.

Oesterreicher, Wulf (2010b): „Linguistik und Interdisziplinarität - Kultur und Sprache bei den Pirahã in der selva amazônica“, in: Romanistisches Jahrbuch 61, 67-101.

Oesterreicher, Wulf (2014): „Sprachwissenschaft und Philologie im Horizont der Geschichtlichkeit von Sprache und Text“, in: ders./Maria Selig (Hg.), Geschichtlichkeit von Sprache und Text. Philologien - Disziplingenese - Wissenschaftshistoriographie, Paderborn, Fink, 287-330.

Oesterreicher, Wulf (2015): „Zum Status der romanistischen Linguistik - gegen ihre ,Verächter““, in: Michael Bernsen/Elmar Eggert/Angela Schrott (Hg.), Historische Sprachwissenschaft als philologische Kulturwissenschaft in der Romanistik. Festschrift für Franz Lebsanft zum 60. Geburtstag, Bonn, Bonn University Press, 9-22.

Oesterreicher, Wulf (2016): „30 Jahre ,Sprache der Nähe - Sprache der Distanz‘. Zu Anfängen und Entwicklung von Konzepten im Feld von Mündlichkeit und Schriftlichkeit“, in: Helmuth Feilke/Mathilde Hennig (Hg.), 13-77.

Perlmutter, David M. (1978): „Impersonal passives and the unaccusative hypothesis“, in: Proceedings of the 4th Annual Meeting of the Berkeley Linguistics Society, 157-189.

Poplack, Shana/St-Amand, Anne (2009): „Les Récits du français québécois d'autrefois: reflet du parler vernaculaire du $19^{\mathrm{e}}$ siècle“, in: Canadian Journal of Linguistics/Revue canadienne de linguistique 54, 511-546. 
Rizzi, Luigi (1997): „The Fine Structure of the Left Periphery“, in: Liliane Haegeman (Hg.), Elements of Grammar, Dordrecht, Kluwer, 281-337.

Seiler, Guido (2015): „Syntactization, analogy, and the distinction between proximate and ultimate causations“, in: Aria Adli/Marco García García/Göz Kaufmann (Hg.), System, Usage, and Society, Berlin/New York, de Gruyter, 239-263.

Selig, Maria (2017): „Plädoyer für einen einheitlichen, aber nicht einförmigen Sprachbegriff: Zur aktuellen Rezeption des Nähe-Distanz-Modells“, in: Romanistisches Jahrbuch 68, 114-145.

Söll, Ludwig (31985): Gesprochenes und geschriebenes Französisch, Berlin, Schmidt.

Stark, Elisabeth (2015): „La grammaire normative face à la réalité d'usage - l'accord du participe passé dans les corpus oraux du français contemporain“. Keynote bei den Journées PFC an der Universität Wien, 17./18.Juli 2015.

Stark, Elisabeth (2016a): „Relative Clauses“, in: Adam Ledgeway/Martin Maiden (Hg.), The Oxford Guide to the Romance Languages, Oxford, Oxford University Press, 1029-1040.

Stark, Elisabeth (2016b): „Is past participle agreement in French also a normative artefact?“, Vortrag im Research seminar in linguistics an der Universität Genf, 10. Mai 2016.

Stark, Elisabeth (2017): „Pertinence de l'analyse grammaticale en linguistique variationnelle“, in: Langage \& Société 160/161, 267-281.

Stark, Elisabeth/Riedel, Isabelle (2012): „L'accord du participe passé dans les SMS francophones du corpus SMS suisse“, in: Romanistisches Jahrbuch 63, 116-138.

Stempel, Wolf-Dieter (1988): „Die schwierige Einheit der romanischen Philologie“, in: Fritz Nies/ Reinhold R. Grimm (Hg.), Ein ,unmögliches Fach‘. Bilanz und Perspektiven der Romanistik, Tübingen, Narr, 41-58.

Zribi-Hertz, Anne (2011): „Pour un modèle diglossique de description du français: quelques implications théoriques, didactiques et méthodologiques“, in: Journal of French Language Studies 21/1, 231-256. 